\title{
Artelogie
}

Recherche sur les arts, le patrimoine et la littérature de I'Amérique latine

16 | 2021

Fotografía y migraciones, siglos XIX-XXI.

\section{Témoin oculaire de la guerre : photographies du soldat Hermann E. et le travail de la mémoire}

\section{Méri Frotscher}

Traducteur : Jaqueline Rocha

\section{OpenEdition}

\section{Journals}

\section{Édition électronique}

URL : https://journals.openedition.org/artelogie/9157

DOI : 10.4000/artelogie.9157

ISSN : 2115-6395

\section{Éditeur}

Association ESCAL

\section{Référence électronique}

Méri Frotscher, « Témoin oculaire de la guerre : photographies du soldat Hermann E. et le travail de la mémoire », Artelogie [En ligne], 16 | 2021, mis en ligne le 27 janvier 2021, consulté le 03 septembre 2021. URL : http://journals.openedition.org/artelogie/9157 ; DOI : https://doi.org/10.4000/artelogie. 9157

Ce document a été généré automatiquement le 3 septembre 2021

Association ESCAL 


\title{
Témoin oculaire de la guerre : photographies du soldat Hermann E. et le travail de la mémoire
}

\author{
Méri Frotscher
}

Traduction : Jaqueline Rocha

\section{Introduction}

1 Cet article 1 analyse des photographies de guerre qui font partie d'un album de famille organisé par Hermann E., au service de l'armée allemande pendant la Seconde Guerre mondiale. L'album m'a été montré par son fils Horst lors d'un entretien en 2009 pour un projet de recherche sur le retour en Allemagne de citoyens allemands résidant au Brésil, comme sa famille, dans les années $1930 .{ }^{2}$ Ce qui a le plus retenu mon attention pendant l'entretien, c'est la narration saisissante qu'il a faite des expériences de son père sur le front russe en 1941. Bien qu'il n'ait lui-même que quatre ans, en 1941, des épisodes impliquant son père en URSS ont été racontés avec tant de détails, comme si lui, son fils, était présent aux moments dont on se souvenait.

Lors de ce premier entretien, il m'avait déjà montré un album de photos de famille qui contenait également des photos prises par son père pendant la guerre, y compris sur les fronts de l'Ouest et de l'Est, et le petit journal que son père avait écrit sur un carnet de notes lors de sa participation au front russe. Il s'agissait de deux récits, l'un visuel et l'autre écrit, de sa participation à la Seconde Guerre mondiale. Le fait d'écrire un journal et de prendre des photos avec son propre appareil photo, dans la précarité du front, montre à lui seul qu'il était conscient de participer d'un événement de grande importance, non seulement pour sa propre histoire, mais également pour l'histoire du monde.

3 Le journal commence avec le début de la campagne militaire contre l'URSS et couvre donc la période allant de juin 1941 à janvier 1942, année où il a été blessé et retiré du front. Malgré les conditions difficiles des marches et des combats, il a trouvé le temps de faire des enregistrements presque tous les jours dans un petit carnet de notes et de prendre des photos avec son appareil photo. Les notes, rédigées au crayon en allemand, sont très informatives, succinctes et parfois 
télégraphiques. Le sommeil, la fatigue et les activités épuisantes l'empêchaient de réfléchir plus longuement aux faits dont il était à la fois protagoniste et témoin.

4 La première annotation reconnaissable sur le carnet de notes fait référence au 27 juin 1941, cinq jours après le début de l'opération Barbarossa. La mention de certaines villes le long des 42 pages permet au lecteur de tracer de façon approximative, le chemin parcouru. En se basant sur ce qu'écrit Hermann et sur l'histoire de la guerre, on peut découvrir qu'il faisait partie du groupe qui se dirigeait vers Moscou par le centre (Heeresgruppe Mitte). ${ }^{3}$

Dans cet article, je me concentre sur l'analyse des " photographies de guerre » d'Hermann Edlich, leur disposition et leur présentation dans l'album. Je vais essayer d'établir, dans les limites de cet article, des liens avec ce qui apparaît dans le journal.

6 Dans l'analyse qui suit, je ne m'attarderai pas sur toutes les pages contenant des photographies prises pendant la guerre, mais seulement sur quelques pages plus importantes permettant de décoder des aspects du processus de création du soldat/photographe et de comprendre le processus de signification du passé à travers les légendes qui identifient les images.

\section{Photographies de guerre et travail de la mémoire}

En 1922, à l'âge de 11 ans, Hermann émigre au Brésil avec sa famille de la région de la Ruhr, en Allemagne. La famille s'est installée dans une région rurale de la haute vallée d'Itajaí, dans l'État de Santa Catarina, où elle vivait de l'agriculture. Dans sa jeunesse, Hermann devient cordonnier et en 1938, déjà marié et père du journaliste interrogé, Horst, il rentre en Allemagne avec sa petite famille dans l'espoir d'améliorer la situation économique, sous l'influence de la propagande nazie. ${ }^{4}$

$8 \quad$ Les photographies de l'album présentées lors de l'interview avec son fils Horst couvrent la période de 1920, alors qu'Hermann vivait encore en Allemagne, jusqu'en 1950, prises après son retour avec sa femme et ses enfants au Brésil. La plupart des photographies datent des années 1930 et 1940, lorsque sa famille vivait en Allemagne. Cet album contient des images de trois générations et montre comment les migrations entre l'Allemagne et le Brésil, et inversement, ainsi que les migrations intra-régionales internes au Brésil et les déplacements provoqués par la guerre en Europe ont également fait partie de l'histoire visuelle de la famille.

Dans cet article, je me concentrerai uniquement sur les photos de guerre, lorsque le soldat Hermann a été envoyé dans différentes régions de l'Allemagne, de Pologne, de France, de Belgique et de l'URSS. Elles constituent 17 des 56 pages de l'album de Hermann. Même si ce n'est pas le seul album, le fait que ces photos aient été rangées avec des photos de famille montre à quel point la guerre a ainsi été inscrite dans la mémoire familiale. Il n'est donc pas difficile de comprendre pourquoi l'histoire de Hermann à la guerre est une partie importante de l'interview que j'ai faite avec son fils Horst, sur la période vécue en Allemagne (entre 1938 et 1948), quand il avait entre 1 et 11 ans.

Un élément qui attire l'attention dans l'observation de l'album est qu'il existe trois types de légendes, indiquant au moins deux interventions supplémentaires dans l'album après son organisation. Il est également à noter que les légendes de certaines photos de guerre sont plus explicatives que les photos de famille et, dans plusieurs d'entre elles, il établit un dialogue en allemand avec ses petits-enfants, car il s'identifie lui-même comme "Opa" (grand-père). Certaines d'entre elles ont des phrases entières formulées pour ceux qui regardent l'album, 
témoignant ainsi l'intention d'expliquer les faits à la famille, qui n'a pas connu la guerre sur le front.

11 Les albums de famille peuvent être compris comme des moyens par lesquels la mémoire culturelle de ce groupe social peut être constituée. Le spécialiste de la mémoire Jan Assmann, qui a développé les notions de mémoire culturelle et de mémoire communicative - deux formes différentes de mémoire collective -, explique que la première est une mémoire collective à long terme basée sur un ensemble de textes, d'images et de rites constamment répétés. Selon lui, c'est grâce au maintien et à la répétition des mémoires que l'image de soi d'un groupe social est stabilisée et informée (ASSMANN, 2008, p. 109-118). Je pense qu'une telle répétition, dans le cas d'un album photo, se fait par son observation continue et ses explications au fil du temps.

Comme le fait remarquer Roswitha Becker, "lors de la composition d'un album de famille, des moments photographiquement fixes de personnes à un moment précis ou dans des événements uniques sont reliés afin de créer une image d'une biographie et d'une famille " (BRECKNER, 2014, 303). Ils sélectionnent, organisent et donnent un sens aux images du passé. Ces considérations nous permettent de réaliser que non seulement les faits, mais aussi les interprétations du passé vécu peuvent être basées sur des souvenirs créés et figés dans des albums de photographies. C'est dans ce sens que l'historien Alistair Thomson fait également référence à l'album photo, une forme autobiographique populaire par laquelle les gens associent les images et les textes pour représenter une version de la vie familiale et de l'identité (THOMSON, 2011, p. 3898).

Si les photographies sont des "actes de témoignages oculaires", ce sont des témoins silencieux, comme le souligne Peter Burke (2004, p. 17-18), et c'est à l'historien d'utiliser des méthodes et des questions pour les interroger à partir du concept que les photographies sont des documents, et non le passé lui-même.

Dans ce texte, je ne prends pas la photo comme un miroir de la réalité, mais comme une "représentation à partir du réel » et en même temps comme un "document du réel " (source historique), en fonction de la matérialité de l'enregistrement dans le temps et dans l'espace (KOSSOY, 1999, 31). Comme le met en garde Boris Kossoy, expert en photographie, la photographie a sa propre réalité, car bien qu'il existe une "première réalité", qui est le passé lui-même, il y a aussi la réalité du document, la réalité de la représentation. C'est cette " seconde réalité » construite par la photographie qui doit être décodée dans l'analyse (KOSSOY, 1999, p.22). Notre ensemble de photographies montre les différentes compositions et comportements $d u$ photographe. Si dans les images qui font référence au début de la guerre, quand les Allemands gagnent encore, Hermann se comporte souvent comme un touriste, - il prend des photos d'églises et d'autres bâtiments représentatifs, il insère dans l'album des cartes postales d'Allemagne du Nord, de Pologne et de France occupées, - ce positionnement change ou du moins prend un sens différent lorsque l'on découvre le front russe, comme on le verra plus tard.

On peut dire qu'en général, la plupart des photographies de guerre sont disposées en série dans l'album, sans respecter un ordre chronologique. Il y a aussi des photos prises séparément d'autres périodes et lieux et des cartes postales au milieu. Les premières datent de la fin de la carrière militaire, prises dans le Nord de l'Allemagne, alors qu'Hermann était déjà invalide pour le front; suivies des photos prises en URSS, puis les cartes postales, les photos prises en Allemagne en tant que soldat/touriste; puis des séries de photos en ordre chronologique inverse, d'abord celles prises en URSS, puis en France et en Belgique, et enfin en 1940 lors de son entraînement militaire. Il est à remarquer qu'après la première organisation de l'album, quelques photos prises séparément, très différentes, ont été incluses au milieu de certaines séries. 

mais pas impossible, d'observer comme il enregistre le déroulement de la guerre. Les photos prises sur le front de l'Ouest révèlent une vie quotidienne plus calme, voire agréable. Sur les photos prises plus tard, en URSS, les conséquences matérielles et humaines de la guerre apparaissent.

18 J'analyserai ici quelques séries de photographies dans l'ordre chronologique des faits. Je commence par les photographies prises en Prusse orientale, où s'est déroulé son entraînement militaire pour devenir soldat, avant d'être envoyé sur le front de l'Ouest. Il avait alors 29 ans et n'avait effectué aucun service militaire au Brésil, probablement parce qu'il n'avait pas été naturalisé. Cette série se trouve sur les trois dernières pages de l'album. J'ai choisi la dernière page afin de réfléchir à la composition individuelle des photographies, à la façon dont le soldat y apparaît, à la composition de l'ensemble de photos et à la manière dont il s'identifie lui-même au moyen d'une légende.

Sur la page étaient collées des photographies qui documentaient des scènes vécues dans la caserne. Dans la plupart d'entre elles, il est représenté seul ou avec d'autres soldats.

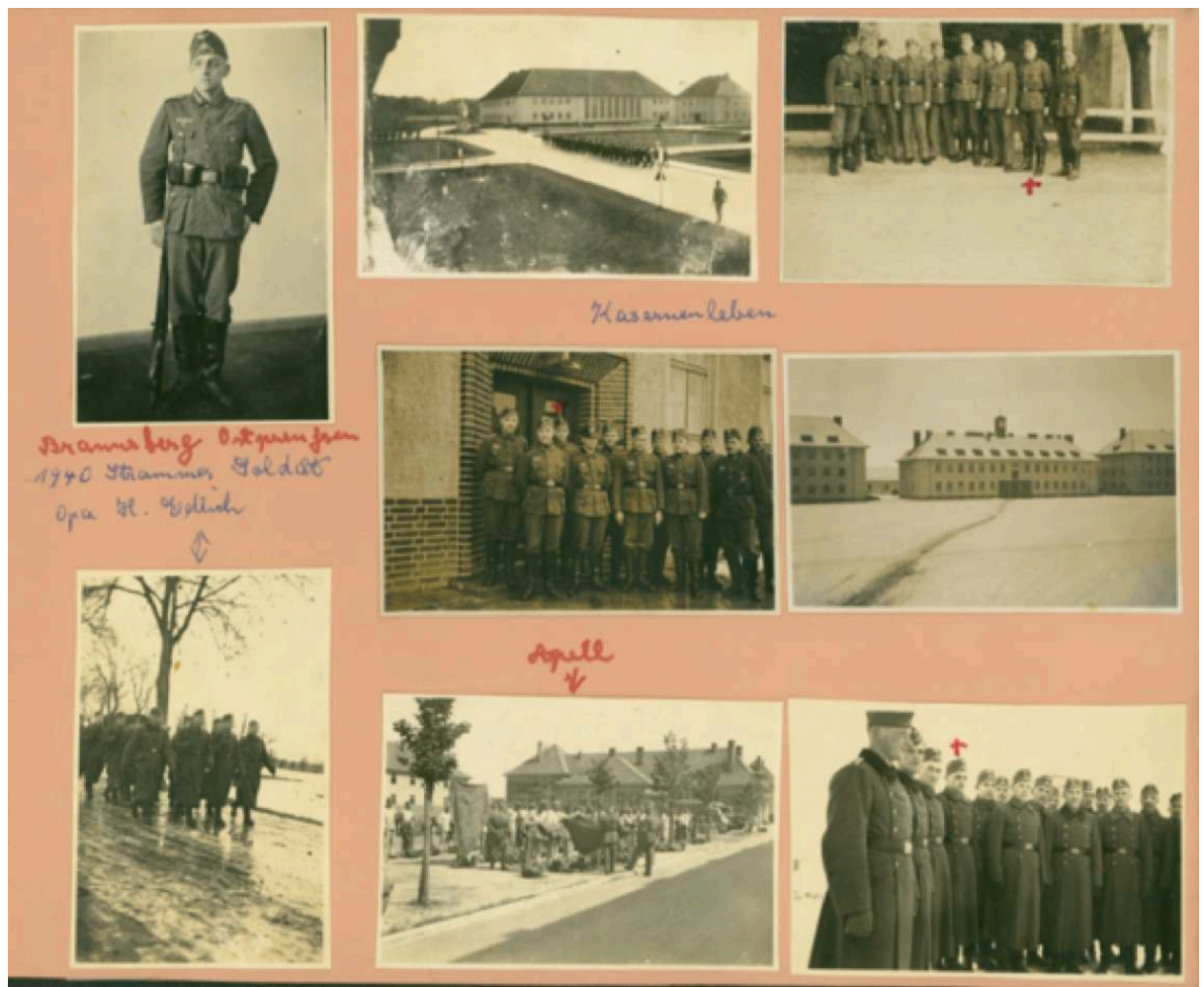

Figure 1: Page 56 de l'album photo d'Hermann E.

Les sujets sur les photographies sont: des soldats en pose pour photo, impeccables en uniforme dans plusieurs types d'uniformes, y compris lui, seul; des soldats en marche; les soldats passés en revue (qu'il identifie par le mot «Apell»); les installations de la caserne, photographiées en plan panoramique. Ce sont des scènes de la vie quotidienne, qu'il résume dans la légende comme "la vie à la caserne ». En regardant la composition générale sur la page, on voit Hermann intégré et subordonné à l'institution de l'armée allemande.

21 Sur la photo de corps entier où il apparaît seul, il est en uniforme, armé et dans une position de garde-à-vous. C'est ainsi qu'il a écrit une annotation à l'encre bleue : "1940 Soldat en position de garde-à-vous. Grand-père H. Edlich ». Il établit ainsi une relation entre le passé - le jeune soldat de l'époque - et le présent, lui au moment d'écrire la légende, déjà grand-père. Une deuxième 
légende, en rouge, indique l'emplacement de la caserne de Braunsberg, en Prusse orientale. À l'encre rouge, il se marque d'une croix sur les photographies dans lesquelles il apparaît avec d'autres soldats.

Sur les photos, les soldats semblent ordonnés et vêtus de façon impeccable. Comme son fils Horst l'a déclaré dans un entretien, à l'époque, son père était alors persuadé que la guerre serait de courte durée. ${ }^{5}$ Il aurait eu l'espoir que l'Allemagne regagnerait des territoires perdus après la défaite dans la Première Guerre mondiale.

Les photographies de guerre suivantes font référence chronologiquement à sa participation sur le front de l'Ouest, en Belgique et surtout en France. L'analyse des photographies de l'une des pages reproduites ci-dessous, avec l'observation de la sélection des sujets, des «cadres» et des moments photographiés, explique le but/intentionnalité du photographe. ${ }^{6}$

Le sujet prédominant de 14 des 15 photographies est l'occupation de la France en 1940. Elles témoignent le sentiment de faire partie d'un tournant historique en faveur de l'Allemagne. Comme le dit Hermann lui-même dans les légendes, elles montrent la visite de membres de son unité militaire à l'endroit où l'armistice franco-allemand a été signé en 1918, et à proximité du bâtiment qui leur servait de cantonnement.

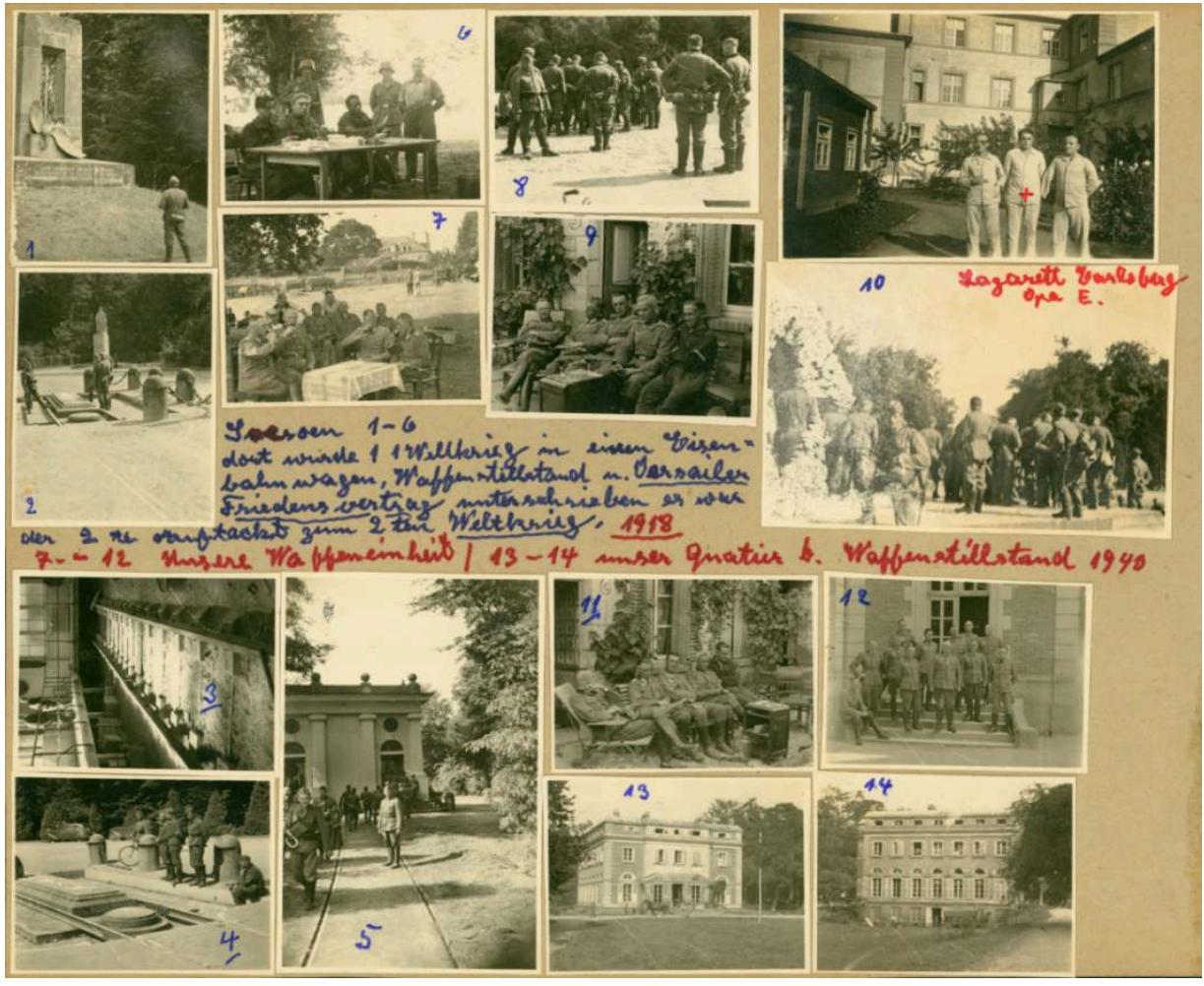

Figure 2 : Page 49 de l'album photo d'Hermann E.

Dans quatre des scènes photographiées à Soissons (1 à 4), se trouvent des soldats de son unité militaire qui observent des monuments français en mémoire de la victoire de la France lors de la Première Guerre mondiale. Parmi eux, se trouve le monument érigé en 1922 dans la "Clairière de l'Armistice", à Compiègne, en mémoire de la signature de l'armistice de paix en faveur de la France dans ce même lieu, le 11 novembre 1918. Le monument montre une sculpture de l'« Aigle germanique " abattu par le "Glaive français ». Les négociations et la signature de l'armistice, qui a scellé la défaite allemande, avaient eu lieu dans un wagon de train, qui est devenu plus tard connu sous le nom de " wagon de l'armistice . $^{8}$ 

de photographier des officiers en train de trinquer autour d'une table en plein air, dans les locaux du bel immeuble occupé (photo 7) ou en pose devant le même bâtiment (photos 9, 11 et 12). Sur une photo de la page suivante, Hermann lui-même apparaît autour d'une table, avec d'autres soldats, en train de prendre un déjeuner de fête (il utilise l'expression "Festessen ») en plein air dans les jardins du château. L'idée de célébrer un banquet entre les soldats est renforcée par une autre photo, prise uniquement sur la table.

31 Revenant à la page précédente de l'album, je voudrais également souligner la présence de légendes bicolores pour les photos. La première, précédemment citée, a été écrite en bleu au centre de la page et fait référence aux 6 premières photos. Les inscriptions en rouge ont été faites plus tard, donnent des informations complémentaires sur les autres photos au lecteur/spectateur de l'album, qu'il imaginait être ses petits-enfants : «1918 » indique l'année du traité de Versailles ; les autres informations expliquent que les photos 7 à 12 proviennent de son unité militaire (Waffeneinheit); que celles des numéros 13 et 14 représentent le bâtiment qui a servi à cantonner les soldats ; que celle de numéro 10 le représente, identifié comme «Opa H. E. » dans le Lazarett Carlsberg. La dernière photographie mentionnée, dans laquelle il apparaît en pyjama du lazarett, semble y avoir été collée plus tard, faute de place dans l'album, car elle s'écarte du sujet des 
autres photographies. Elle a été faite près de deux ans plus tard, alors qu'il était invalide de guerre et à une époque où l'excitation vécue en 1940 s'était déjà en partie dissipée, comme on peut le voir dans le journal écrit après.

Une autre série de photographies fait référence au front russe. Là aussi, son regard est marqué par la perspective idéologique allemande de la guerre. Les photos ont comme centre de l'image des villages brûlés (par les Russes), des huttes "primitives» de paysans russes, des églises transformées en silos. Les destructions causées par la guerre sont associées aux Russes, comme dans la légende de la page 45 (figure 3) : «C'est toujours pareil, les combats, les lieux brûlés par les Russes, seule une cheminée apparaît, parfois une église ». Ainsi, il documente la stratégie de la «terre dévastée » utilisée par les Russes.

Les photos renforcent une vision anticommuniste, présente de manière plus explicite dans le journal. Dans cet aspect, je souligne à nouveau le processus de création du photographe, marqué idéologiquement, car, comme le souligne Kossoy, «les données du réel enregistrées dans les photographies correspondent à un produit documentaire élaboré culturellement, techniquement et esthétiquement, et donc idéologiquement : rgistre/creation " (KOSSOY, 1999 : p. 34-35). Comme le souligne également Peter Burke, bien que la photographie puisse être considérée comme un " art documentaire ", comme un témoignage de la réalité, elle n'a jamais un "regard innocent ", c'est-à-dire "sans attentes ni préjugés d'aucune sorte » (BURKE, 2004 : p.24).

Dans le journal, écrit en même temps que la prise des photographies en URSS, il y a plusieurs passages dans lesquels Hermann exprime l'anticommunisme et des aspects du "nationalisme quotidien", une vision nationale du monde, à l'aide de laquelle les soldats allemands ont également produit des images sélectives de l'Europe de l'Est (MÜLLER, 2007 : p. 176). Dans l'un des registres de Poloszk, Hermann observe d'une manière ironique et anticommuniste la situation de la population et de l'économie locale : "Les gens d'ici sont très pauvres, comme partout ailleurs ici, des produits industriels misérables dans le paradis rouge ". ${ }^{11}$

Cependant, certaines de ses photographies montrent aussi les premières difficultés rencontrées par les troupes allemandes en URSS : chars et voitures embourbées, tombes improvisées de soldats allemands, marches interrompues. Ces photos apparaissent juxtaposées à des images de la vie quotidienne du front vécue par lui et les autres soldats, telles que celles collées sur la page ci-dessous, qui montrent la préparation de la nourriture (ironisée par le mot "Privatküche", cuisine privée), le paysage vu pendant leur marche (les photos se concentrent sur un lieu brûlé par les Russes, des chevaux morts, des tombes des soldats, un canon russe), des prisonniers de guerre faits par sa division. Comme les combats n'ont pas pu être enregistrés instantanément, une partie de leurs résultats - comme l'arrestation d'ennemis - sont enregistrés plus tard par la caméra. Le fait qu'Hermann soit apparu enregistré en photos, seul ou avec ses "camarades", montre qu'il voulait être présent. D'autres ont utilisé son appareil photo, probablement pour répondre à sa demande, comme dans la troisième photo de la première ligne. 


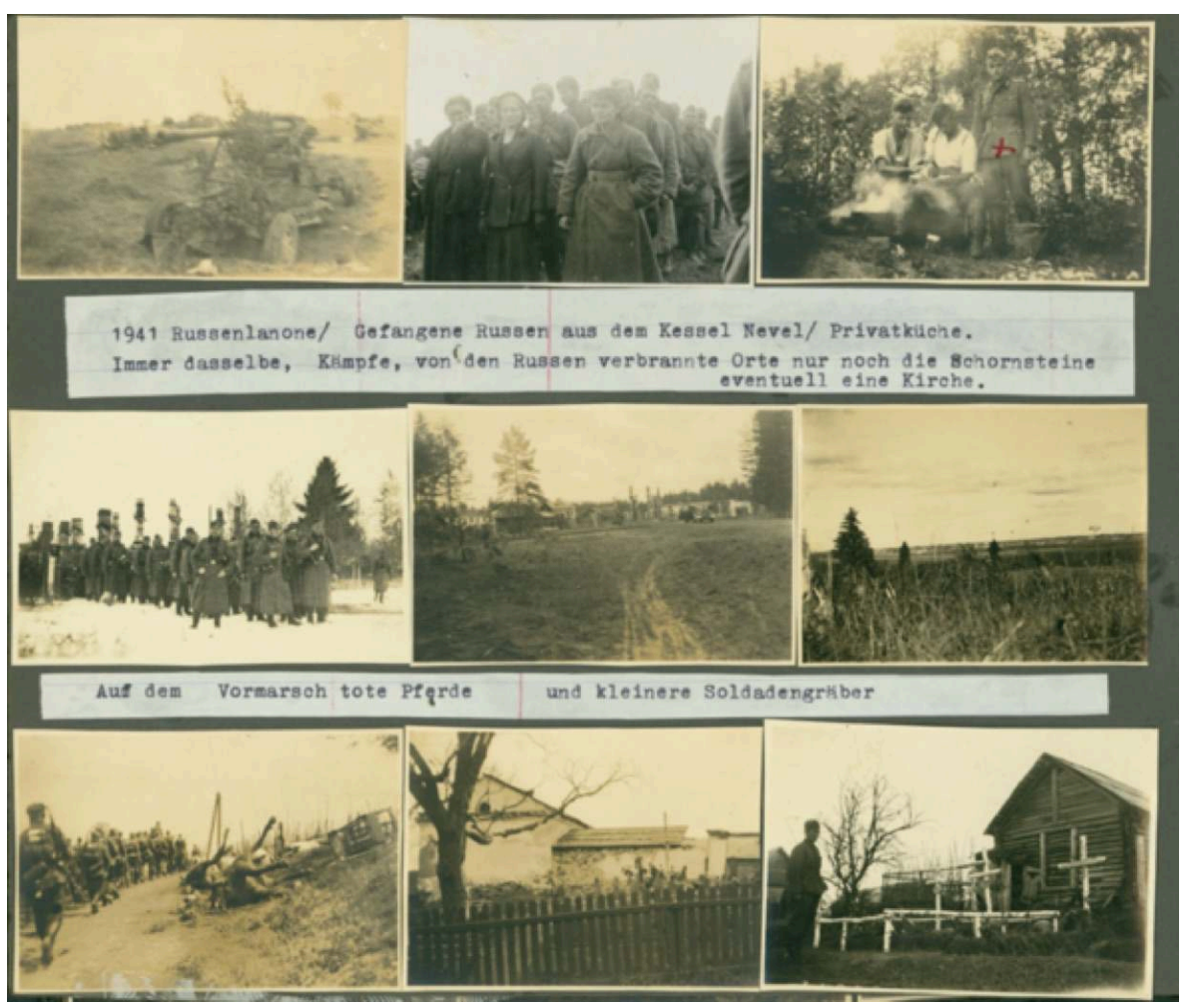

Figure 3 : Page 45 de l'album photo d'Hermann E. ${ }^{12}$

Dans la deuxième photo ci-dessus, de gauche à droite, les prisonniers pris lors du siège de Nevel sont concentrés. Trois femmes russes apparaissent au premier plan devant d'autres prisonniers masculins. La participation des femmes russes aux combats a été un fait qui a impressionné les soldats allemands. Si le livre impressionnant, dans lequel l'écrivain Svetlana Alexijevich documente la participation des femmes russes à la Seconde Guerre mondiale est intitulé " La guerre n'a pas un visage de femme" (ALEXIJEVICH, 2016) dans la photographie ci-dessus, la perspective du photographe permet de voir même l'expression de leurs visages. Mais à part celleci, seules deux autres photos documentent les femmes russes. Dans son récit visuel de la guerre, la prédominance est masculine.

Dans le registre du 23-07-1941, il mentionne ce qui suit dans son journal : «[...] De 10h à 18h, patrouilles forestières et ramassage de butin, de nombreux prisonniers, aussi des femmes. 15-20 $\mathrm{km}$. $21 \mathrm{~h}$ départ de la marche vers Biwak $3 \mathrm{~km} .{ }^{13}$ L'importance des réalisations de l'armée allemande, $y$ compris le nombre de prisonniers incarcérés, est surtout soulignée dans d'autres écritures du journal. Dans l'album de photos cela est visible dans la légende de la page 47 (figure 4), où il figure une autre photo de prisonniers (troisième de gauche à droite, deuxième ligne), au milieu des photographies (1 à 4) qui attestent "l'installation d'un Bunker à Nevel pour le siège des armées russes [;] là, 3600000 prisonniers russes ont été capturés ».

La photographie d'une partie des prisonniers assiégés montre, au premier plan, l'un d'eux debout avec un masque à gaz sur la tête. Il regarde le photographe. Derrière lui se trouvent des prisonniers assis dos à la scène photographiée, mais certains inclinent la tête vers la photo. À côté et devant eux (à l'arrière-plan de la photo) d'autres hommes se tiennent, certains en civil, d'autres en uniforme, parmi lesquels un officier allemand. Nombreux sont ceux qui observent le sujet au premier plan en train d'être photographié. Ce n'est pas seulement l'aspect morbide du masque qui attire l'attention ici, mais aussi l'acte même de photographier l'un des prisonniers 
dans cette situation. L'intention n'est pas claire (moquerie ?), ni comment l'image a été composée (probablement que le photographe n'a pas mis le masque volontairement).

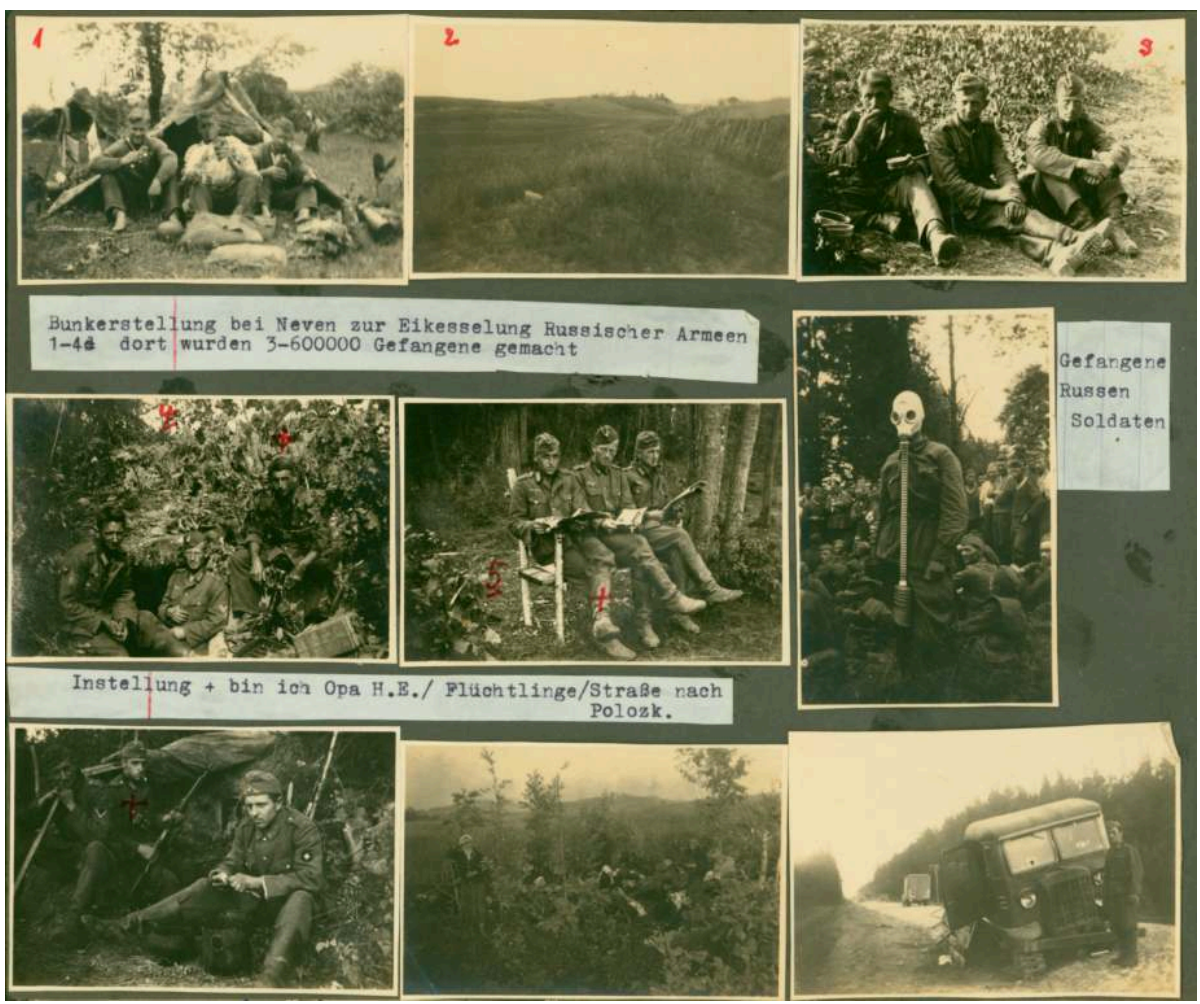

Figure 4 : Page 47 de l'album photo d'Hermann E. ${ }^{14}$ photographier, aux côtés d'autres "camarades » de combat, l'une pendant un repas, l'autre en simulant la lecture, une autre avec un fusil à la main, en position de combat, comme il écrit dans la légende, devant l'abri. Le marquage avec une croix sur son image - ce qui se produit également dans d'autres photographies - indique sa présence dans la composition des scènes photographiées. La façon dont il s'identifie dans la légende - "En position +, c'est moi, grandpère S.E. » - démontre une fois de plus pour qui il avait créé l'album : ses petits-enfants.

40 Même si le photographe simule des situations vécues, les photos ne peuvent exprimer les sentiments ressentis lors des combats, les souffrances résultant des luttes, le froid extrême, les marches sans fin. Il ne se concentre pas non plus sur les images de cadavres humains, qu'ils soient allemands ou russes. Ceci est rapporté dans le journal, bien que de manière concise, ce qui démontre dans divers passages sa peur de la mort. Les scènes photographiées, comme on peut le voir, ont été choisies en fonction de son point de vue sur ce qu'il considérait pertinent à enregistrer. Comme le souligne l'historien Alistair Thomson, même si les photographies nous permettent de connaître ou de nous rappeler le passé plus clairement, elles peuvent filtrer sélectivement la mémoire. (THOMSON, 2011 : p. 3861).

41 Les photos de guerre qui suivent chronologiquement, disposées sur une seule page, peuvent se résumer à des moments photographiés dans le lazarett, déjà de retour en Allemagne, et à l'école militaire. En janvier 1942, ses deux orteils avaient gelé à cause du froid extrême, raison pour laquelle Hermann fut transporté sur un traîneau avec d'autres soldats blessés par une mine.

Les légendes de cette dernière série de photographies illustrent plus explicitement le processus continu de réinterprétation du passé, puisque l'on peut percevoir au moins trois moments de l'écriture. Ici, il est clair que les premières légendes ont été dactylographiées, puis ajoutées avec 
des informations manuscrites en bleu, puis en rouge. L'écriture manuscrite est plus interprétative que celle qui a été dactylographiée. Tout indique qu'avec le passage du temps et l'avancement de l'âge, le besoin d'apporter des informations à l'album semble s'être accru, et peut-être même des justifications par rapport à la génération des petits-enfants. Face au fait que le sens n'est pas figé sur la photographie, parce qu'il est toujours ouvert à la lecture de celui qui la voit, il semblait vouloir éviter que les photographies, avec le temps, perdaient leur capacité à "dire» quelque chose sur son passé personnel ou qu'il ait voulu, lui-même, imprimer son interprétation.

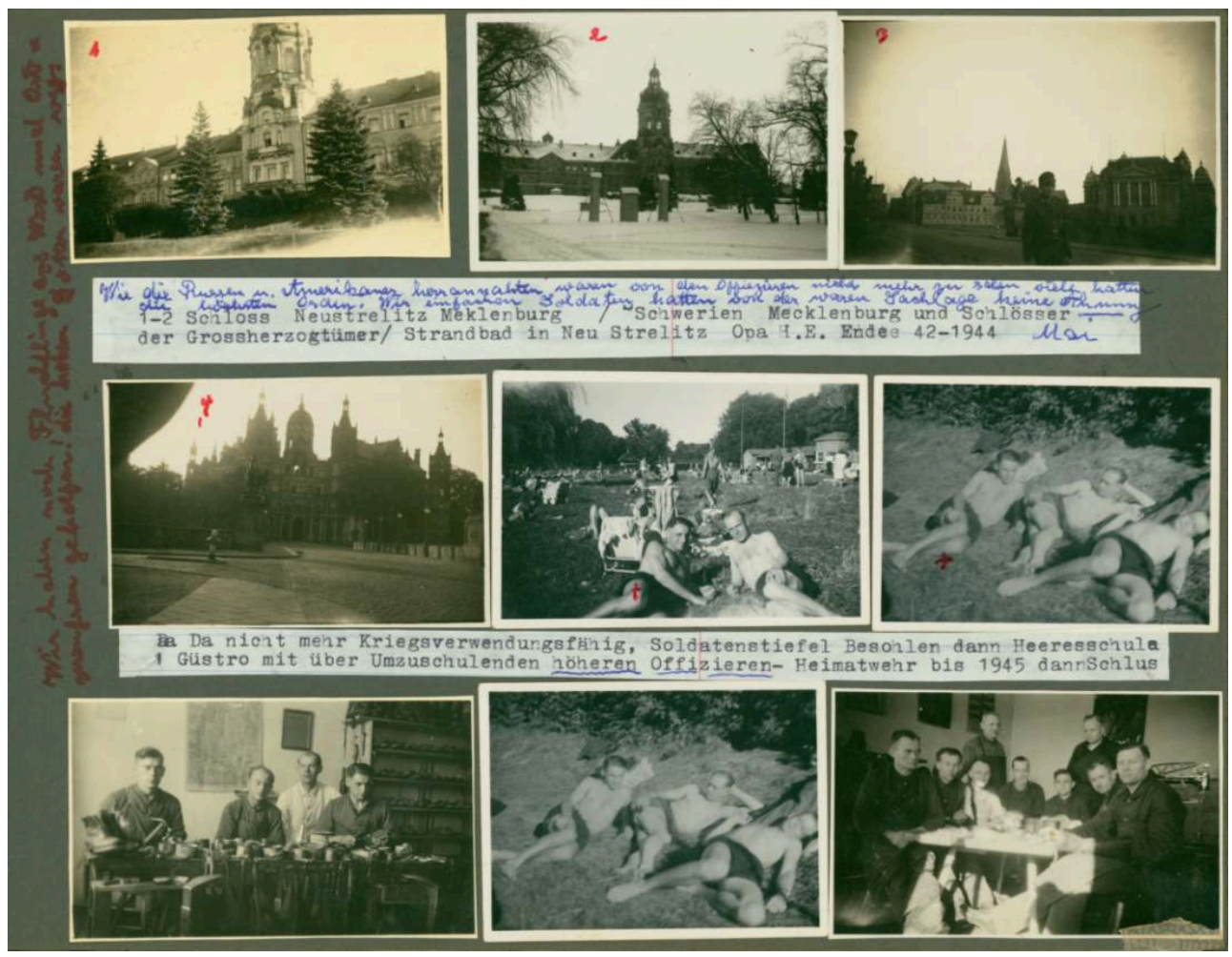

Figure 5 : Page 47 de l'album photo d'Hermann E.

43 La séquence de photographies composée sur la page ci-dessus fait référence à la période postérieure à 1942. On peut voir des images panoramiques de châteaux dans l'État de Mecklembourg, au Nord de l'Allemagne, où Hermann était au service de l'armée. Sur l'une d'elles, il pose à nouveau comme un touriste. Après des photos de lui et de ses collègues prenant un bain de soleil et exerçant le métier de cordonnier et d'officiers qui, comme lui, étaient en cours de réadaptation professionnelle. Deux légendes dactylographiées informent les lieux et les années des photos et de leurs activités jusqu'à la fin de la guerre, toujours avec l'auto-identification «Opa H. E.» (Grand-père H.E.). Dans la seconde légende, il retrace ainsi sa trajectoire jusqu'en 1945 : «Ne pouvant plus être employé à la guerre, réparation de semelles de bottes de soldats, puis l'école militaire à Güstro [sic] avec des officiers supérieurs pour apprendre un nouveau métier. Heimatwehr jusqu'en 1945. Alors, c'est fini ». Par le mot Heimatwehr ("défense de la patrie »), il représente son rôle à la fin de la guerre. ${ }^{15}$ Dans cette même légende dactylographiée, il a plus tard souligné, avec un stylo bleu, les mots "officiers supérieurs ". Avec le même stylo, il ajoute la critique suivante, au-dessus de la première légende dactylographiée : "Quand les Russes et les Américains se sont approchés, on n'a plus rien vu des officiers (allemands), beaucoup d'entre eux avaient les ordres militaires les plus élevés. Nous, simples soldats, nous n'avions pas la moindre idée de l'état des choses ». Cette légende est en désaccord avec la plupart des légendes, étant donné qu'elle ne fournit pas seulement des informations. À travers elle, il 
interprète les événements du passé et exprime à la fois une critique morale des officiers et un ressentiment de leur attitude à l'égard des "simples soldats». Une autre légende écrite du bas vers le haut, maintenant en lettres rouges, dans le coin gauche de la page, renforce le contenu de la légende en bleu: "Nous aidons toujours les réfugiés de Prusse occidentale et orientale! Les grands dieux étaient partis. » À travers la métaphore sarcastique "les grands dieux », il critique à nouveau l'attitude des officiers, contrastant ici avec l'attitude de solidarité qui le sépare des autres soldats, qui "aidaient » encore les réfugiés allemands avant l'arrivée des Russes. Le mot "encore" renforce cette opposition et l'image de soi d'un soldat solidaire envers les Allemands civils en fuite.

Le fait qu'Hermann n'ait pas été fait prisonnier de guerre a été raconté par son fils dans son interview. À bicyclette, Hermann traversa une grande partie de l'Allemagne, du Mecklembourg à la Bavière, jusqu'à ce qu'il puisse rejoindre sa famille, qui y avait été évacuée en 1943. L'imminence de l'arrivée des troupes ennemies et la défaite ont fait tomber l'ordre établi jusquelà, c'est-à-dire la relation de subordination et de hiérarchie entre les membres de l'armée allemande. Après la guerre, Hermann trace une ligne de démarcation entre les " grands dieux »les hauts fonctionnaires - et les "simples soldats", alors qu'il s'auto-identifie. Il est important d'ajouter qu'Hermann, qui occupait le poste de sergent sur le front russe, avait été rétrogradé au poste de simple soldat après un acte d'insubordination survenu peu avant qu'il ne soit blessé et retiré du front..$^{16}$

Ces légendes manuscrites révèlent l'intention d'établir des liens avec des expériences vécues qui ne sont pas apparentes sur les photographies. En observant la photo avec les officiers, il s'en tient à ce " détail », au "punctum» (ce qui me punit dans une photographie, comme le dit Roland Barthes) (BARTHES, 1984: 68), lui faisant dénoncer par écrit ce qui serait arrivé après l'acte photographié, lorsque les officiers ont abandonné les soldats. À cet égard, il est important de prendre en compte le pouvoir que la photographie possède de raviver le passé, de réveiller quelque chose chez le spectateur, comme l'a souligné Barthes.

Ces légendes manuscrites sont donc le résultat d'un processus de réflexion ultérieure sur leur place et leur action dans l'armée et d'un ressentiment visible envers les officiers. À cet égard, il convient de préciser le sens psychanalytique du ressentiment. Comme le formule la psychanalyste Maria Rita Kehl, le ressentiment peut être compris comme un «mécanisme de défense de soi ", quand on attribue à autrui la responsabilité de ce qui nous fait souffrir. Pour cette raison, "la version imaginaire du manque, dans le ressentiment ", est toujours interprétée comme une perte. (KEHL, 2004, p.11). Hermann servit toute la guerre dans cette armée, dont les "grands dieux ", comme il l'appela plus tard, étaient les représentants. Après la guerre, il a dû évaluer les gains et les pertes liés au retour en Allemagne et, par conséquent, il est resté au service de l'armée pendant presque toute la guerre dans des conditions extrêmes, lorsqu'il combattait sur le front russe. Ironiquement, le cordonnier qui avait décidé de quitter le Brésil pour améliorer son statut social et économique a vécu la fin de la guerre et la période qui a suivi, en Bavière, de la même profession pratiquée au Brésil. Mais cette fois avec les orteils des pieds mutilés.

\section{Considérations finales}

Dans cet article, j'ai essayé de montrer non seulement comment Hermann a représenté sa participation à la Seconde Guerre mondiale d'une manière visuelle, mais aussi comment la mémoire a fonctionné dans l'interprétation des événements actuels de l'écriture des légendes. Dans ce processus, le ressentiment imprègne le récit de sa participation à la guerre. 
Les photographies de guerre présentes dans l'album couvrent différentes temporalités, liées à la production, au collage et à l'identification de photographies. Le photographe, et parfois aussi celui qui a été photographié, qui a composé un tableau pour ses souvenirs à partir de ce qu'il a vécu pendant les cinq années de guerre, du travail que sa mémoire a exercé tout au long de sa vie et de l'interaction avec les lecteurs/spectateurs attendus de l'album: les petits-enfants. Cette observation montre que les photographies ne sont pas indépendantes du temps et ne sont pas figées dans leurs sens. Comme le soulignent les historiens Alexander Freund et Alistair Thomson, de même que l'histoire orale, la photographie n'est pas seulement une évidence, elle exige un "travail de mémoire" et constitue une forme de mise en récit. (FREUND \& THOMSON, 2011 : 311).

A partir du moment où Hermann assemble l'album, il devient spectateur de ses propres photos (et de celles prises par d'autres), compositeur d'un récit visuel et écrit, à travers l'arrangement des photographies et leur description. Sa participation à la Seconde Guerre mondiale est représentée et réinterprétée pour la génération des ses petits-enfants, qui ont vécu non seulement à une époque distincte, mais également dans un espace géographique différent, au Sud du Brésil. C'est à partir de cette distance temporelle et géographique qu'il donne un sens à certaines photographies par l'écriture. Cependant, les photographies sont là, elles sont ouvertes aux sens, elles sont sa perspective de sa participation à la Seconde Guerre mondiale.

\section{BIBLIOGRAPHIE}

ALEXIJEVICH, Svetlana. A guerra não tem rosto de mulher. São Paulo: Cia. das Letras, 2016.

ASSMANN, J. 2008. Communicative and cultural memory. In: A. ERLL; A. NÜNNING (ed.), Cultural Memory Studies: An international and interdisciplinary handbook. Berlin, New York, de Gruyter, p. 109-118.

BARTHES, Roland. A câmara clara: Nota sobre a fotografia. Rio de Janeiro: Editora Nova Fronteira, 1984.

CHALFEN apud BRECKNER, Roswitha. Narrar, mostrar, ver. Como analisar um álbum de fotos de família em conexão com uma entrevista narrativa. Civitas, Porto Alegre, v. 14, n. 2, p. 285-306, mai.-ago. 2014.

FREUND, Alexander; THOMSON, Thomson. Introduction: Oral History and Photography. In: (Ed.) Oral History and Photography. New York: Palgrave Macmillan, 2011.

BURKE, Peter. Testemunha ocular: história e imagem: Bauru: Edusc, 2004.

ECHTERNKAMP, Jörg. Der Zweite Weltkrieg: Die 101 wichtigste Fragen. München, Verlag C. H. Beck, 2010.

FROTSCHER, Méri. Narrar a vida durante o III Reich. Interpretação de "trajetórias de vida" escritas por "retornados" à Alemanha a partir do Brasil. Naveg@merica, Murcia, v. 11, p. 1-18, 2013. 
. „Als Nationalsozialist tat ich jederzeit unter schwersten persönlichen Opfern meine Pflicht“. Autobiographische Erzählung eines Rückwanderers aus Brasilien im institutionellen Kontext. Bios, Leverkusen, v. 26, p. 129-143, 2014.

. Memórias do nazismo e da guerra em duas gerações de descendentes de alemães repatriados para o Brasil. Fronteiras, Florianópolis, v. 26, p. 23-49, 2015.

KAMMER, Hilde; BARTSCH, Elisabet. Nationalsozialismus: Begriffe der Zeit der Gewaltherschaft 1933-1945. Reinbek bei Hamburg: Rowohlt Taschenbuch Verlag, 1992.

KEHL, Maria Rita. Ressentimento. 3a. Ed. São Paulo: Casa do Psicólogo, 2004.

KOSSOY, Boris. Realidades e ficções na trama fotográfica. Cotia: Ateliê Editorial, 1999.

MÜLLER, Sven Oliver. Deutsche Soldaten und ihre Feinde: Nationalismus an Front und Heimatfront im Zweiten Weltkrieg. Frankfurt a. Main: S. Fischer, 2007.

THOMSON, Alistair. Family Photographs and Migrant Memories: Representing Women's Lives. In: FREUND, Alexander; THOMSON, Alistair (Ed.) Oral History and Photography. New York: Palgrave Macmillan, 2011. Ebook.

\section{NOTES DE FIN}

1. La recherche qui a abouti à cet article est financée par le CNPq (Bourse Productivité en Recherche et Appel Universel MCTIC/CNPq nº 28/2018). Titre du projet; «Des tropiques au Reich: vivre et raconter le retour du Brésil en Allemagne nazie ».

2. Projet de recherche "Mémoires de la guerre et du nazisme: le rapatriement des citoyens brésiliens d'Allemagne après la Seconde Guerre mondiale ", développé entre 2009 et 2011.

3. Le groupe était commandé par le maréchal Fedor von Bock, qui dirigeait 49 divisions. Disponible à l'adresse https://de.wikipedia.org/wiki/Heeresgruppe_Mitte Consultation du 27-06-2018.

4. Au sujet du retour de citoyens allemands du Sud du Brésil en Allemagne sous le régime nazi, voir, entre autres publications de l'auteur : FROTSCHER (2013; 2014 ; 2015).

5. Entretien réalisé par l'auteur avec Horst E. Indaial, Santa Catarina, Brésil, le 16 juillet 2009.

6. Kossoy dresse la liste de ces étapes et d'autres étapes de la création photographique dans sa discussion sur le processus de création du photographe : KOSSOY, idem, p. 26.

7. Les inscriptions en bas du monument étaient dédiées aux «soldats héroïques de la France/ défenseurs de la patrie et de la glorieuse droite/des libérateurs glorieux d'Alsace et de la Lorraine. » https://www.guerre1914-1918.fr/le-monument-a-larmee-francaise-liberatrice-delalsace-lorraine-compiegne-60-oise/ Consultation le 10-08-2018.

8. Le wagon avait été transformé en bureau du commandant en chef Ferdinand Foch pendant la guerre.

9. Le wagon est devenu un monument à la victoire française sur l'Allemagne, lorsqu'en 1921 il a été transporté au Musée de l'Armée à Paris, où il est resté exposé jusqu'en 1927. Il fut ensuite restauré et exposé au musée Clairière de l'Armistice à Compiègne. Ce n'est pas un hasard si Hitler ordonna, le 22 juin 1940, de retirer le wagon du bâtiment et 
de le placer au même endroit dans la Clairière de Compiègne pour la signature de l'armistice en faveur de l'Allemagne. Voir ECHTERNKAMP (2010 : p. 28-29) et https:// de.wikipedia.org/wiki/Wagen_von_Compi\%C3\%A8gne Consultation du 10-08-2018.

10. Toutes les traductions des références en allemand ont été faites librement par l'auteur.

11. À l'original : « Bevölkerung hier sehr arm wie hier überall, Industrieerzeugnisse im roten Paradies unterm Hund». Enregistrement concernant le 14-07-1941. Journal de Hermann E. p. 07.

12. Traduction des légendes : " 1941 Canon russe/Prisonniers russes du siège Nevel/ cuisine privée »; " Toujours la même chose, les combats, les lieux brûlés par les Russes, seule une cheminée apparaît, parfois une église "; "Les chevaux morts près de la rangée des soldats en marche et les petites tombes des soldats ».

13. À l'original : «[...] Ab 10.0 bis 18.0 Wald abkämmen und Beute sammeln, viele

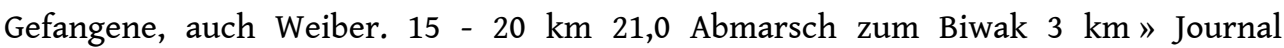
d'Hermann E. p. 12.

14. Traduction des légendes : «Abris près de Neven pour le siège des armées russes. 1-4 il y a eu 3-600000 Russes capturés "; «Les soldats russes faits prisonniers "; «En position + c'est moi Opa H. E./ Réfugiés / Autoroute vers Polozk ».

15. L'expression est similaire au Heimatfront, utilisé par le gouvernement nazi pour caractériser le rôle de la population allemande pendant la guerre. Ainsi, le front de guerre lui-même (Kriegsfront) était associé à un front interne. Dans : KAMMER \& BARTSCH, 1992, p. 88-89.

16. D'après son journal (16-12-1941) et une interview de son fils : journal de guerre de Hermann E. ; entretien réalisé par l'auteur avec Horst E. Indaial - SC, Brésil, 16 juillet 2009.

\section{RÉSUMÉS}

Cet article analyse des photographies de guerre faisant partie d'un album de famille organisé par Hermann E., qui a servi l'armée allemande pendant la Seconde Guerre mondiale. Emigré enfant au Brésil en 1922, il rentre en Allemagne avec sa nouvelle famille en 1938, où il est appelé à servir dans l'armée allemande peu après le déclenchement de la guerre. Le texte est centré sur l'analyse des photographies, leur mise en page et leur présentation dans l'album. Le but est de décoder les aspects du processus de création du soldat/ photographe et de percevoir le processus de signification du passé pour la famille au moyen des légendes.

Este artigo analisa fotografias de guerra que fazem parte de um álbum de família organizado por Hermann E., o qual serviu ao Exército Alemão durante a Segunda Guerra Mundial. Nascido em 1911 e emigrado ainda criança para o Brasil, em 1922, em 1938 ele retornou com sua recém-formada família para a Alemanha. Ali ele foi convocado para servir ao Exército Alemão logo após o início da guerra. 0 foco do texto é a análise das fotografias, de sua disposição e de sua apresentação no álbum. O objetivo é decodificar aspectos do processo de criação do soldado/fotógrafo e perceber o processo de significação do passado por meio de sua disposição no álbum e das legendas. 
INDEX

Mots-clés : mémoire; photographie; album de famille ; Seconde Guerre mondiale; Allemagne; Brésil

Palavras-chave : memória; fotografia; álbum de família; Segunda Guerra Mundial; Alemanha; Brasil

\section{AUTEURS}

\section{MÉRI FROTSCHER}

Université d'État de l'Ouest de Paraná. Doctorat en Histoire Culturelle de l'Université Fédérale de Santa Catarina, au Brésil. Professeure agrégée du Collège d'Histoire du Centre des Sciences Humaines, de l'Éducation et des Lettres de l'Université d'État de l'Ouest de Paraná. Boursière du CNPq - Conseil National du Développement Scientifique et Technologique. 